\section{Proposta de um modelo de avaliação da atenção pública à saúde bucal da criança}

\author{
Proposal of a model for the evaluation of public \\ oral health care for children
}

\author{
Propuesta de un modelo de evaluación de la \\ atención pública a la salud oral de niños
}

\section{Resumo}

O objetivo deste estudo foi propor um modelo para a avaliação da qualidade da atenção à saúde bucal da criança. O marco conceitual para a elaboração da matriz avaliativa foi composto por estudos de avaliação de serviços de atenção à saúde bucal no Brasil e em outros países, e levantamento e revisão das políticas públicas vigentes relacionadas à saúde da criança no Brasil. A qualidade da gestão municipal na atenção básica da saúde bucal infantil foi avaliada sob duas dimensões: Gestão e Provimento. Na primeira, foram dispostas duas subdimensões: articulação e recursos, e na segunda, mais duas subdimensões: promoção e prevenção, e diagnóstico e tratamento. A matriz de avaliação ficou composta por nove indicadores na dimensão de Gestão e nove na dimensão de Provimento. Os indicadores foram elaborados em oficinas com especialistas. O modelo tem condições de ser aplicado em municípios com diferentes portes populacionais, e permite identificar os pontos nos quais os gestores devem aperfeiçoar suas ações.

Avaliação em Saúde; Saúde Bucal; Crianças
Luiz Gustavo Teixeira Martins 1

Josimari Telino de Lacerda 2

Daniela Alba Nickel 1

Jefferson Traebert 1 


\section{Introdução}

A qualidade dos serviços de saúde é objeto de atenção e preocupação por parte da sociedade brasileira. A busca pela qualidade dos serviços de assistência à saúde no Brasil tem recebido grande destaque do Ministério da Saúde. Isso se dá pela importância da adequação das políticas públicas às necessidades da população e do retorno adequado dos investimentos, que se reflete na melhoria da situação de saúde do país ${ }^{1}$.

A avaliação é um importante instrumento de gestão em saúde. Possibilita a identificação de aspectos débeis e a indicação de medidas para correção de rumos e alcance de metas. No Brasil, as atividades avaliativas no âmbito da atenção básica em saúde geralmente são pontuais. O Estado de Santa Catarina é considerado um caso exemplar de avaliação em saúde porque vem desenvolvendo um processo de institucionalização da avaliação da gestão e de programas na Atenção Básica e na Saúde Bucal 2. O processo participativo de avaliação adotado naquele estado busca assegurar que a visão de diferentes atores seja considerada na análise do objeto de estudo, de forma a garantir a apreensão das diversas dimensões da realidade 2 .

Avaliar e monitorar os resultados alcançados em relação à organização e provisão dos serviços e possíveis impactos na saúde da população são questões fundamentais em um cenário de grande investimento e expansão da rede na Atenção Básica 3. O desafio colocado à gestão de saúde pública constitui na utilização de ferramentas metodológicas de avaliação e monitoramento que permitam obter conhecimento sobre as políticas, programas e serviços, e que levem em consideração as particularidades do programa, já que ele poderá apresentar resultados diferentes quando implantado sob diferentes contextos 4 . A avaliação em saúde deveria ser realizada como prática sistemática para fornecer aos gestores as informações necessárias para a intervenção, já que muitas informações registradas nos serviços acabam não sendo utilizadas para o planejamento e tomada de decisões. As informações coletadas poderiam constituir uma base importante para o monitoramento contínuo das ações de saúde 5 . Assim, a avaliação dos serviços de saúde pode ser entendida como um meio de prover a melhoria contínua da atenção com equilíbrio de custos, gerando um atendimento de qualidade para o usuário 1 .

A avaliação de serviços de atenção à saúde bucal foi proposta por Chaves ${ }^{6} \mathrm{em} \mathrm{1986}$. Narvai 7 define atenção à saúde bucal como um conjunto de ações de alcance coletivo, que tem como objetivo manter a saúde oral, incluindo a assistência.
Tais ações vão além do ambiente da odontologia e do próprio setor da saúde, contemplando ações em todos os setores, desenvolvidas no conjunto da comunidade, como emprego, saneamento e educação. Para Pinto 8 a qualidade no contexto da saúde bucal pode ser entendida como sinônimo de melhores serviços e de êxitos nas iniciativas e projetos do setor. Apesar dos autores 6,8 referirem, há bastante tempo, a necessidade de estudos de avaliação dos serviços odontológicos prestados à população, há uma grande carência de pesquisas nesta área. Colussi \& Calvo ${ }^{1}$ ao procederem a uma revisão da literatura sobre o tema identificaram somente 17 trabalhos na área. Concluíram que a dificuldade, somada à escassez, de encontrá-los e selecioná-los relacionava-se à utilização de descritores de assuntos referentes à avaliação em diferentes contextos e situações. Uma nova revisão de artigos publicados sobre avaliação em saúde bucal no período de 2011-2015 nas bases de dados SciELO e PubMed trouxe 23 trabalhos. Após uma seleção por título e resumo, oito artigos permaneceram para a leitura completa do texto. Desses, sete tratavam da avaliação da saúde bucal na atenção básica e um de avaliação na atenção de média complexidade, sobre centros de especialidades odontológicas. No entanto, quando verificados os juízos de valor, somente um artigo apresentou esta informação.

Assim, diante da necessidade de estudos que avaliem a qualidade da atenção à saúde bucal prestada à população em geral, é importante salientar as significativas mudanças do comportamento epidemiológico das principais doenças bucais, notadamente as quedas dos indicadores de cárie dentária em populações infantis. Entretanto, observa-se que os grupos em desvantagem social apresentam os piores indicadores, o que é explicado pelos determinantes sociais do processo saúde-doença ${ }^{9}$. Diante disso, o objetivo deste trabalho é propor um modelo para avaliação da qualidade da atenção pública à saúde bucal infantil.

\section{Métodos}

O modelo elaborado foi parcialmente baseado no modelo desenvolvido por Colussi \& Calvo ${ }^{1}$ no Núcleo de Extensão e Pesquisa em Avaliação em Saúde (NEPAS) da Universidade Federal de Santa Catarina (UFSC). O modelo teórico-lógico foi a base para a elaboração de uma matriz avaliativa valendo-se da definição de dimensões e subdimensões advindas de um marco conceitual. $\mathrm{O}$ marco conceitual para a elaboração da matriz avaliativa foi composto por: revisão da literatura 
para busca de estudos de avaliação de serviços de atenção à saúde bucal no Brasil 3,10,11,12,13,14, $15,16,17,18,19,20,21$ e levantamento e revisão das políticas públicas vigentes relacionadas à saúde da criança no Brasil, como a Política Nacional de Promoção de Saúde 22 e de Saúde Bucal 23, Programa Saúde na Escola 24, Estatuto da Criança e do Adolescente (ECA) 25 e a Constituição Federal de 1988.

A definição das dimensões, subdimensões, indicadores e medidas da matriz avaliativa foi objeto de quatro oficinas de trabalho com pesquisadores e professores vinculados ao NEPAS, UFSC e Universidade do Sul de Santa Catarina (UNISUL). O tipo de oficina utilizado foi técnica mista, que inicialmente usou o Comitê Tradicional, em que houve uma discussão aberta entre os especialistas, e por último utilizou a oficina online. Na primeira oficina do Comitê, foi apresentado o modelo teórico-lógico e o marco teórico utilizado para a sua elaboração. Na segunda e terceira foram realizadas discussões para a definição dos indicadores e medidas. As sugestões para mudanças na matriz, indicadores ou medidas, foram propostas e discutidas por intermédio da oficina on-line. Para tanto, foram encaminhadas correspondências eletrônicas com a matriz avaliativa e o projeto em anexo para um grupo de sete especialistas. Para cada indicador definiu-se uma medida, estabelecendo-se a fonte dos dados para o seu cálculo.

Finalizada essa etapa, procedeu-se o teste de aplicabilidade do modelo. Os dados primários foram provenientes das Secretarias Municipais de Saúde, com base em um instrumento de coleta de dados on-line, utilizando-se o software FormSUS (http://formsus.datasus.gov.br/site/ default.php). Uma amostra não probabilística de 18 municípios, sendo dois por macrorregião, obedeceu às seguintes etapas: foram criados grupos de municípios por macrorregiões e porte populacional de acordo com os dados do Departamento de Informática do SUS (DATASUS), organizados em ordem decrescente por tamanho da população residente no ano de 2013, e divididos em quartis. Em cada macrorregião de saúde foi selecionado o primeiro município do primeiro quartil e o último município do quarto quartil.

Todos os dados coletados foram inseridos em planilha eletrônica no Microsoft Excel (Microsoft Corp., Estados Unidos). Os resultados das medidas dos indicadores foram convertidos para escores 0,1 ou 2, em que 0 indicou situação Insatisfatória, 1 situação Regular e 2 situação Satisfatória. A determinação do juízo ocorreu segundo a distribuição quartílica, sendo os $25 \%$ melhores colocados considerados Satisfatório, os $50 \%$ intermediários considerados Regular e os $25 \%$ piores colocados classificados como Insatisfatório. Após a conversão e cálculo dos indicadores, os escores foram somados para cada município e para cada indicador. Com essa pontuação estabeleceu-se um ranking com base nos valores atingidos. Valendo-se dos valores finais apresentados pelos municípios, chegou-se a um juízo de valor para a qualidade da atenção em saúde bucal infantil prestada. A determinação para soma final dos juízos de valor de cada município e de cada indicador teve os seguintes pontos de corte: Insatisfatório: soma dos escores até 20; Razoável: soma maior que 20 e menor que 31; e Satisfatório: soma maior ou igual a 31 .

Este estudo foi realizado em conformidade com a Resolução no 466/2012 do Conselho Nacional de Saúde e foi submetido e aprovado pelo Comitê de Ética em Pesquisa da UNISUL sob protocolo no 792.298 .

\section{Resultados}

O marco teórico que sustenta o modelo teóricológico do presente processo avaliativo está baseado na legislação vigente do Sistema Único de Saúde (SUS) e do ECA. O primeiro norteia os princípios fundamentais e a organização dos serviços de saúde, dos quais são originadas as políticas de saúde para promover a saúde e reduzir riscos e danos à saúde bucal da criança. O segundo aborda tratamento social e legal que deve ser oferecido às crianças e adolescentes do Brasil, com um espírito de maior proteção e cidadania. A segunda parte foca o conhecimento atual sobre evidências científicas acerca de práticas diagnósticas, preventivas e terapêuticas na atenção à saúde da criança que os serviços podem dispor para promover a saúde bucal, diagnosticar e tratar as doenças bucais neste segmento da população (Figura 1).

A matriz avaliativa foi composta por 2 dimensões, 4 subdimensões e 18 indicadores, sendo 9 indicadores para cada dimensão. A Tabela 1 mostra as dimensões, subdimensões e indicadores.

Na Tabela 2 estão apresentados os juízos de valor no teste de aplicabilidade do modelo de avaliação, atribuídos aos municípios catarinenses referente aos dados do ano de 2013. A cor verde significa que o município obteve um resultado Satisfatório em um determinado indicador, a amarela Regular e a cor vermelha Insatisfatório. O valor máximo que o município pode alcançar é 36 , já que são 18 indicadores que podem receber valores de 0 (cor vermelha, Insatisfatório), 1 (cor amarela, Regular) ou 2 (cor verde, Satisfatório).

Em relação à soma dos escores de cada indicador, a maioria apresentou um resultado 


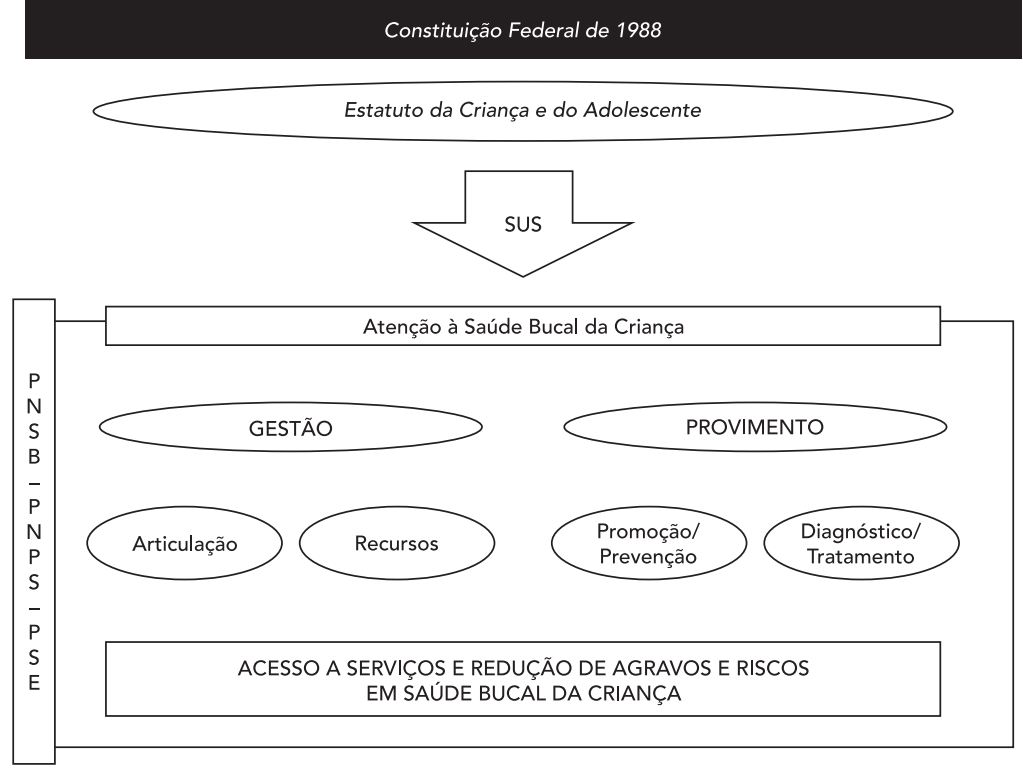

PNPS: Política Nacional de Promoção da Saúde; PNSB: Política Nacional de Saúde Bucal; PSE: Programa Saúde na Escola; SUS: Sistema Único de Saúde.

Regular. Dos 18 indicadores, 10 somaram de 21 a 30. Os indicadores que tiveram a maior soma (32) foram: planejamento adequado do tratamento (código E), tratamento completado (TC) (código G) e desenvolvimento de hábitos saudáveis (código J), que têm, respectivamente, como medidas: total de 1a consulta programática em crianças, no ano/total da população de crianças; número total de TC em crianças, no ano/número total de 1a consulta programática em crianças, no ano; e \% escolas municipais com controle de alimento na cantina.

Já a assistência especializada (código F) que tinha como medida a existência de referência operante para a especialidade de Odontopediatria, foi o pior indicador, com soma igual a 18, e foi o único indicador classificado como Insatisfatório (menor que 21). Os sete indicadores restantes ficaram classificados como Satisfatórios, com a soma maior que 30 .

As duas dimensões obtiveram resultados similares, com apenas cinco pontos de diferença entre a soma dos seus escores. A dimensão Provimento obteve o melhor resultado. Nessa dimensão, os melhores indicadores foram planejamento adequado do tratamento (código E) e tratamento completado (código G). Já na dimensão da Gestão, o pior indicador foi planejamento de recursos humanos (código M) e aporte de recursos extras para a saúde bucal (código Q), e na dimensão do Provimento ficou com o pior indicador a assistência especializada (código F).

O resultado da soma que constituiu o juízo de valor atribuído aos municípios estudados concentrou-se em grande parte no resultado Satisfatório. Dos 18 municípios, quase a metade (8) obteve uma soma maior que 30. Florianópolis (código 51) foi o único que conseguiu o valor máximo de 36. Somente 4 municípios apresentaram resultado Insatisfatório (menor que 21) e os outros 6 municípios, resultado Regular (de 21 a 30). O município com o pior resultado foi Presidente Castello Branco (código 14). Os municípios de grande porte populacional (códigos com final 1) conseguiram melhores resultados que os de pequeno porte (códigos com final 4), com uma diferença de 6 pontos na soma dos escores. Em relação às macrorregiões, o Meio Oeste e Grande Oeste registraram as piores somas, e a região da Grande Florianópolis e Foz do Rio Itajaí, os melhores resultados. 
Matriz avaliativa, dimensões, subdimensões e indicadores.

\begin{tabular}{|c|c|}
\hline Dimensão/Subdimensão & Indicadores \\
\hline \multicolumn{2}{|l|}{ Gestão } \\
\hline \multirow[t]{3}{*}{ Articulação } & Desenvolvimento de hábitos saudáveis \\
\hline & Acesso ao flúor \\
\hline & Vigilância do teor de flúor na água de abastecimento \\
\hline \multirow[t]{6}{*}{ Recursos } & Planejamento dos recursos humanos \\
\hline & Acesso ao cirurgião-dentista \\
\hline & Adequação da equipe de saúde bucal \\
\hline & Adequação da capacidade instalada \\
\hline & Aporte de recursos extras para saúde bucal \\
\hline & Adequação da estrutura física \\
\hline \multicolumn{2}{|l|}{ Dimensão/Subdimensão } \\
\hline \multicolumn{2}{|l|}{ Provimento } \\
\hline \multirow[t]{4}{*}{ Promoção/Prevenção } & Educação em saúde bucal \\
\hline & Prevenção individual \\
\hline & Saúde bucal na escola \\
\hline & Ações integradas na equipe de saúde \\
\hline \multirow[t]{5}{*}{ Diagnóstico/Tratamento } & Planejamento adequado do tratamento \\
\hline & Assistência especializada \\
\hline & Tratamento completado \\
\hline & Acesso ao tratamento \\
\hline & Equidade \\
\hline
\end{tabular}

\section{Discussão}

A elaboração de modelos de avaliação voltados para as gestões municipais constituem ferramentas de acompanhamento e qualificação do desenvolvimento de ações para implementar a qualidade da atenção prestada. Justifica-se a sua construção pela necessidade de desenvolver um modelo específico e apropriado para a avaliação dos serviços, que poderá ser aplicada em outros momentos e circunstâncias para observar diferentes contextos de atenção à saúde bucal. Ajuda também os gestores e profissionais na identificação de problemas e no desenvolvimento de planejamento e mecanismos para as diferentes ações 26,27 . Os estudos sobre avaliação da saúde bucal têm o potencial de trazer as respostas para esses questionamentos, gerando maior interesse sobre avaliações de ações e serviços em saúde bucal.

Na dimensão Gestão, os indicadores mais bem colocados foram: desenvolvimento de hábitos saudáveis, acesso ao cirurgião-dentista e adequação da estrutura física, os demais apresentaram juízo de valor Regular. O desenvolvimento de hábitos saudáveis na dimensão
Gestão apresentou a melhor soma no juízo de valores, este achado corrobora com o estudo de Souza et al. 28 em que os autores afirmam que o incentivo ao desenvolvimento de hábitos saudáveis é um objetivo da promoção da saúde, e incluem o estímulo à alimentação saudável e à prática de exercícios físicos. O controle da alimentação das crianças nas cantinas das escolas municipais é uma articulação intersetorial e de responsabilidade do gestor, por meio de uma Lei Municipal aprovada pela Câmara Municipal ou de uma articulação entre as Secretarias da Saúde e da Educação, para favorecer a aquisição de hábitos alimentares mais saudáveis, que repercute na qualidade da saúde bucal dos escolares. A intersetorialidade em saúde é a relação conhecida entre o setor saúde e outros setores, neste caso o de educação por intermédio da escola, construída para atuar em um tema comum, visando a alcançar resultados de saúde de uma maneira mais efetiva e eficiente 29 .

O avanço significativo do número de cirurgiões-dentistas no serviço público aconteceu após a criação da equipe de saúde bucal (ESB) e inclusão destes profissionais na equipe de saúde da família no ano 2000, dentro de um contexto 
Tabela 2

Resultado do teste de aplicabilidade do modelo de avaliação.

\begin{tabular}{|c|c|c|c|c|c|c|c|c|c|c|c|c|c|c|c|c|c|c|c|c|}
\hline \multirow[t]{3}{*}{ Macrorregião * } & \multirow[t]{3}{*}{ Município } & \multicolumn{18}{|c|}{ Indicadores } & \multirow[t]{3}{*}{ Soma } \\
\hline & & \multicolumn{8}{|c|}{ Dimensão Provimento } & \multicolumn{10}{|c|}{ Dimensão Gestão } & \\
\hline & & A & B & $\mathrm{C}$ & D & $E$ & $\mathbf{F}$ & $\mathbf{G}$ & $\mathrm{H}$ & I & $\mathrm{J}$ & $\mathrm{K}$ & $\mathbf{L}$ & $\mathrm{M}$ & $\mathbf{N}$ & 0 & $\mathbf{P}$ & Q & $\mathbf{R}$ & \\
\hline Grande Florianópolis & 51 & & & & & & & & & & & & & & & & & & & 36 \\
\hline Vale do Itajaí & 31 & & & & & & & & & & & & & & & & & & & 35 \\
\hline Sul & 61 & & & & & & & & & & & & & & & & & & & 35 \\
\hline Nordeste & 71 & & & & & & & & & & & & & & & & & & & 35 \\
\hline Serra Catarinense & 91 & & & & & & & & & & & & & & & & & & & 35 \\
\hline Planalto Norte & 81 & & & & & & & & & & & & & & & & & & & 34 \\
\hline Foz do Rio Itajaí & 41 & & & & & & & & & & & & & & & & & & & 31 \\
\hline Foz do Rio Itajaí & 44 & & & & & & & & & & & & & & & & & & & 31 \\
\hline Grande Florianópolis & 54 & & & & & & & & & & & & & & & & & & & 30 \\
\hline Sul & 64 & & & & & & & & & & & & & & & & & & & 30 \\
\hline Nordeste & 74 & & & & & & & & & & & & & & & & & & & 30 \\
\hline Serra Catarinense & 94 & & & & & & & & & & & & & & & & & & & 30 \\
\hline Planalto Norte & 84 & & & & & & & & & & & & & & & & & & & 28 \\
\hline Vale do Itajaí & 34 & & & & & & & & & & & & & & & & & & & 27 \\
\hline Meio Oeste & 11 & & & & & & & & & & & & & & & & & & & 20 \\
\hline Grande Oeste & 21 & & & & & & & & & & & & & & & & & & & 16 \\
\hline Grande Oeste & 24 & & & & & & & & & & & & & & & & & & & 16 \\
\hline Meio Oeste & 14 & & & & & & & & & & & & & & & & & & & 14 \\
\hline Soma & & 25 & 31 & 30 & 28 & 32 & 18 & 32 & 27 & 31 & 32 & 30 & 30 & 23 & 31 & 29 & 30 & 23 & 31 & \\
\hline
\end{tabular}

Nota: amarela: Regular; verde: Satisfatório; vermelha: Insatisfatório.

Indicadores: A - educação em saúde bucal; B - prevenção individual; C - saúde bucal na escola; D - ações integradas na equipe de saúde; E - planejamento adequado do tratamento; F - assistência especializada; G - tratamento completado (TC); H - acesso ao tratamento; I - equidade; J - desenvolvimento de hábitos saudáveis; $\mathrm{K}$ - acesso ao flúor; L - vigilância do teor de flúor na água de abastecimento; $\mathrm{M}$ - planejamento dos recursos humanos; $\mathrm{N}$ - acesso ao cirurgião-dentista; O - adequação da equipe de saúde bucal; P - adequação da capacidade instalada; Q - aporte de recursos extras para a saúde bucal; $R$ - adequação da estrutura física.

* Para cada Macrorregião existe um município de pequeno porte (final 1), e um município de grande porte (final 4).

político e econômico favorável. O Ministério da Saúde estabeleceu, por meio da Portaria no $1.444^{30}$, o incentivo financeiro para incluir as equipes no Programa Saúde da Família (PSF), com a finalidade de reorganizar a atenção à saúde bucal nos municípios, definir o trabalho voltado para a mudança do modelo de atenção e ampliar o acesso às ações de saúde bucal 31. O indicador acesso ao cirurgião-dentista apresentou resultado Satisfatório, indo ao encontro do objetivo de ampliar o acesso aos serviços odontológicos municipais por meio das ESB naatenção básica.

Quanto ao acesso ao flúor e à vigilância do teor de flúor na água de abastecimento, sabe-se que é reconhecida a capacidade preventiva da fluoretação da água, medida de saúde pública regulamentada por lei desde 1975. Diante dos benefícios dessa ação, diversas entidades e órgãos defendem que a fluoretação das águas de abastecimento público é uma medida ideal de saúde pública para a prevenção da cárie dentária, devido à sua eficácia, segurança e baixo custo ${ }^{32}$. Mesmo depois que o Brasil alcançou os índices preconizados pela OMS em relação ao número de cáries aos 12 anos, sabe-se que a distribuição não é homogênea, muitas regiões ainda apresentam índices bem elevados, o que justifica a necessidade do acesso ao flúor e da Vigilância do teor nas águas de abastecimento 9 . A fluoretação é um método muito seguro e efetivo para a prevenção da cárie dentária, entretanto, alguns aspectos requerem atenção quando da implementação do sistema de fluoretação das águas, como por exemplo, a vigilância sobre este sistema e a verificação da concentração natural de flúor na água da localidade.

Em relação à Adequação da ESB e Adequação da capacidade instalada, devido aos resultados Regulares, pressupõe-se que dos municípios analisados a maioria dos gestores institui 
diretrizes mínimas para a estrutura física e a contratação de auxiliares, aproximando-se de um atendimento eficiente e de qualidade à população. A presença do auxiliar na prestação de serviços odontológicos, além de criar um ambiente físico favorável ergonomicamente, aumenta a produtividade e reduz o estresse do cirurgião-dentista 1 .

Entre as dificuldades encontradas na elaboração do modelo, uma que merece especial atenção é a escassa literatura sobre avaliação em saúde bucal voltada aos serviços e qualidade da atenção à saúde. Outro questão reside no fato de que a avaliação incluindo modelos teóricos está pouco difundida, este tipo de pesquisa pode ser considerado como objeto recente. Uma das limitações do estudo foi o número de municípios participantes, sugere-se que sejam realiza- das pesquisas futuras com municípios de diferentes regiões do país e a criação de um modelo de avaliação para outros ciclos de vida, como a adolescência.

Pode-se concluir que o modelo de avaliação da atenção em saúde bucal infantil proposto, demonstrou ser aplicável em municípios com diferentes características e portes populacionais. Contemplou, com base em um modelo teórico lógico, a gestão em seus aspectos de articulação e de recursos, e a dimensão de Provimento da atenção, focada na promoção da saúde e prevenção de agravos, além do diagnóstico e tratamento das principais doenças bucais. A matriz construída apresenta a possibilidade de futuras mudanças, podendo sofrer adaptações de acordo com as necessidades locais dos municípios.

\section{Colaboradores}

L. G. T. Martins, D. A. Nickel e J. Traebert contribuiram na concepção do projeto, análise e interpretação dos dados, redação do artigo e aprovação final da versão a ser publicada. J. T. Lacerda participou da redação e revisão do artigo.

\section{Agradecimentos}

O trabalho teve como fonte de financiamento: chamada no 10/2012 MCTI/CNPq/MS - SCTIE - DECIT - Pesquisa em Saúde Bucal, Processo no 403016/2012-6. 


\section{Referências}

1. Colussi CF, Calvo MCM. Modelo de avaliação da saúde bucal na atenção básica. Cad Saúde Pública 2011; 27:1731-45.

2. Nickel DA, Calvo MCM, Natal S, Freitas SFT, Hartz ZMA. Desenvolvimento da capacidade avaliativa na gestão da atenção básica: um estudo de caso exemplar em Santa Catarina, Brasil, de 2008 a 2011. Cad Saúde Pública 2014; 30:839-50.

3. Almeida PF, Giovanella L. Avaliação da atenção básica à saúde no Brasil: mapeamento e análise das pesquisas realizadas e/ou financiadas pelo Minis tério da Saúde entre os anos de 2000 e 2006. Cad Saúde Pública 2008; 24:1727-42.

4. Tamaki EM, Tanaka OY, Felisberto E, Alves CKA Drumond Junior M, Bezerra LCA, et al. Metodologia de construção de um painel de indicadores para o monitoramento e a avaliação da gestão do SUS. Ciênc Saúde Coletiva 2012; 17:839-49.

5. Scalco SV, Lacerda JT, Calvo MCM. Modelo para avaliação da gestão de recursos humanos em saúde. Cad Saúde Pública 2010; 26:603-14.

6. Chaves MM. Odontologia social. 3a Ed. São Paulo: Editora Artes Médicas; 1986.

7. Narvai PC. Saúde bucal: assistência ou atenção? Documento elaborado para subsidiar a Oficina do Grupo de Trabalho Saúde Bucal no Sistema Local de Saúde - SILOS. http://www.ccs.ufsc.br/spb/ os3_narvai.pdf (acessado em 10/Dez/2013).

8. Pinto VG. A odontologia no município: guia para organização de serviços e treinamento de profis sionais a nível local. Porto Alegre: Revista Gaúcha de Odontologia; 1996.

9. Coordenação Geral de Saúde Bucal, Ministério da Saúde. Banco de dados da Pesquisa Nacional de Saúde Bucal - Projeto SB. http://dab.saude.gov.br/ CNSB/sbbrasil/ (acessado em 01/Abr/2013).

10. Giovanella L, Lobato LVC, Carvalho AI, Conill EM. Sistemas municipais de saúde e a diretriz da integralidade da atenção: critérios para avaliação. Saúde Debate 2002; 26:37-61.

11. Hartz ZMA, Vieira-da-Silva LM, organizadores. Avaliação em saúde: dos modelos teóricos à prática na avaliação de programa e sistemas de saúde. Rio de Janeiro: Editora Fiocruz/Salvador: EDUFBA; 2005.

12. Narvai PC. Avaliação de ações de saúde bucal. Oficina saúde bucal no SUS. São Paulo: Secretaria de Saúde do Estado de São Paulo; 1996.

13. Contandriopoulos AP, Champagne F, Denis JL, Pineault R. A avaliação na área da saúde: conceitos e métodos. In: Hartz ZMA, organizador. Avaliação em saúde: dos modelos conceituais à prática na análise da implantação de programas. Rio de Janeiro: Editora Fiocruz; 1997. p. 29-47.

14. Casotti E, Contarato PC, Fonseca ABM, Borges PKO, Baldani MH. Atenção em saúde bucal no Brasil: uma análise a partir da avaliação externa do PMAQ-AB. Saúde Debate 2014; 38:140-57.

15. Magalhães BG, Oliveira RS, Gaspar GS, Figueiredo N, Goes PSA. Avaliação do cumprimento de atenção secundária em saúde bucal. Pesqui Bras Odontopediatria Clín Integr 2012; 12:107-12.
16. Oliveira ERSB, Queiróz DM, Oliveira RFR. Ava liação da atenção odontológica no Município de Montes Claros (MG) utilizando o sistema de informações ambulatoriais do Sistema Único de Saúde. Revista Unimontes Científica 2014; 16:3-16.

17. Colussi CF, Calvo MCM. Avaliação da atenção em saúde bucal no Brasil: uma revisão da literatura. Saúde \& Transformação Social 2012; 3:92-100.

18. Chaves SCL, Cruz DN, Barros SG, Figueiredo AL. Avaliação da oferta e utilização de especialidades odontológicas em serviços públicos de atenção secundária na Bahia, Brasil. Cad Saúde Pública 2011; 27:143-54

19. Borghi GN, Vazquez FL, Cortelazzi KL, Guerra LM, Bulgareli JV, Pereira AC. A avaliação do sistema de referência e contrarreferência na atenção secundária em Odontologia. RFO UPF 2013; 18:154-9.

20. Rocha GST, Fontes AM, Pereira SLS, Fonteles DSR Avaliação longitudinal de um programa de saúde bucal para pacientes com deficiência. Clin Lab Res Den 2014; 20:88-95.

21. Austregésilo SC, Leal MCC, Góes PSA, Figueiredo N. Avaliação de serviços odontológicos de urgência (SOU): a visão dos gestores, gerentes e profissionais. Pesqui Bras Odontopediatria Clín Integr 2013; 13:161-9.

22. Secretaria de Atenção à Saúde, Secretaria de Vigilância em Saúde, Ministério da Saúde. Política Nacional de Promoção de Saúde. 3a Ed. Brasília: Ministério da Saúde; 2010. (Série B Textos Básicos de Saúde) (Série Pactos pela Saúde 2006, 7).

23. Coordenação Nacional de Saúde Bucal, Departamento de Atenção Básica, Ministério da Saúde. Diretrizes da Política Nacional de Saúde Bucal. Brasília: Ministério da Saúde; 2004.

24. Ministério da Saúde/Ministério da Educação. Programa Saúde na Escola: manual instrutivo. Brasília: Ministério da Saúde/Ministério da Educação; 2013.

25. Brasil. Estatuto da Criança e do Adolescente (1990). Estatuto da criança e do adolescente: Lei no 8.069, de 13 de julho de 1990, Lei no 8.242, de 12 de outubro de 1991. 3a Ed. Brasília : Câmara dos Deputados, Coordenação de Publicações; 2001. (Série Fontes de Referência. Legislação, 36).

26. Soratto J, Faria EM. O diálogo como alternativa à participação popular e o controle social na saúde. In: Ministério da Saúde; Conselho Nacional de Secretarias Municipais de Saúde, organizadores. Prêmio Sérgio Arouca de gestão participativa: experiências exitosas e trabalhos acadêmicos. Brasília: Ministério da Saúde; 2008. p. 90-3.

27. Felisberto E, Freese E, Natal S, Alves CKA. Contribuindo com a institucionalização da avaliação em saúde: uma proposta de auto-avaliação. Cad Saúde Pública 2008; 24:2091-102.

28. Souza EA, Barbosa Filho VC, Nogueira JAD, Azevedo Júnior MR. Atividade física e alimentação saudável em escolares brasileiros: revisão de programas de intervenção. Cad Saúde Pública 2011; 27:1459-71. 
29. Vasconcelos R, Matta ML, Pordeus IA, Paiva SM. Escola: um espaço importante de informação em saúde bucal para a população infantil. Braz Dent Sci 2001; 4:43-8.

30. Ministério da Saúde. Portaria no 1.444, de 28 de dezembro de 2000. Estabelece incentivo financeiro para reorganização da atenção à saúde bucal prestada nos municípios por meio do Programa de Saúde da Família. Diário Oficial da União 2000; 29 dez.

\section{Abstract}

This article proposes a model for quality evaluation of oral health care in children. The conceptual framework for the evaluation matrix consisted of evaluation studies on oral health care services in Brazil and other countries and a survey and review of prevailing public policies for children's health in Brazil. Two dimensions, Management and Provision, were evaluated in the qua lity of municipal management of pediatric oral health care. Management included two component dimensions, articulation and resources, while Provision included two more, "promotion and prevention" and "diagnosis and treatment". The evaluation matrix consisted of nine indicators in the Management dimension and nine in the Provision dimension. The indicators were elaborated in workshops with specialists. The model proved appropriate for application in municipalities with different population sizes and allows identifying the areas where managers should improve their actions.

Health Evaluation; Oral Health; Child
31. Silva SF, Martelli PJL, Sá DA, Cabral AP, Pimentel FC, Monteiro IS, et al. Análise do avanço das equipes de saúde bucal inseridas na Estratégia Saúde da Família em Pernambuco, Região Nordeste, Brasil, 2002 a 2005. Ciên Saúde Coletiva 2011; 16:211-20.

32. Pauleto ARG, Pereira MLT, Cyrino EG. Saúde bucal: uma revisão crítica sobre programações educativas para escolares. Ciên Saúde Coletiva 2004; 9:121-30.

\section{Resumen}

El objetivo fue proponer un modelo para la evaluación de la calidad de la atención a la salud oral de los niños. El marco conceptual para la preparación de la matriz de evaluación estuvo integrado por los estudios de evaluación de la atención a los servicios de salud bucal en Brasil y otros países, y la encuesta y la revisión de las políticas públicas actuales relacionadas con la salud infantil en Brasil. La calidad de la gestión municipal en la atención primaria de la salud oral de los niños se evaluó en dos dimensiones: Gestión y Disposición. En la primera, se disponen dos subdimensiones: coordinación y recursos, y la segunda tiene dos sub-dimensiones más: promoción y prevención, y diagnóstico y tratamiento. La matriz de evaluación se compone de nueve indicadores del tamaño de Gestión y nueve en el tamaño de la Disposición. Los indicadores fueron desarrollados en los talleres con expertos. El modelo es capaz de ser aplicado a los municipios con diferentes tamaños de población, e identifica los puntos en los que los gerentes deben mejorar.

Evaluación em Salud; Salud Bucal; Niño

Recebido em 20/Jan/2015

Versão final reapresentada em 26/Jun/2015

Aprovado em 01/Jul/2015 